\title{
MEAN CURVATURE 1 SURFACES IN HYPERBOLIC 3-SPACE WITH LOW TOTAL CURVATURE II
}

\author{
WAYNE ROSSMAN, MASAAKI UMEHARA, AND KOTARO YAMADA
}

\begin{abstract}
In this work, complete constant mean curvature 1 (CMC-1) surfaces in hyperbolic 3 -space with total absolute curvature at most $4 \pi$ are classified. This classification suggests that the Cohn-Vossen inequality can be sharpened for surfaces with odd numbers of ends, and a proof of this is given.
\end{abstract}

\section{INTRODUCTION}

This is a continuation (Part II) of the paper [14] (Part I) with the same title. As pointed out in Part I, complete CMC-1 (constant mean curvature 1) surfaces $f$ in the hyperbolic 3 -space $H^{3}$ have two important invariants. One is the total absolute curvature $\operatorname{TA}(f)$, and the other is the dual total absolute curvature $\operatorname{TA}\left(f^{\#}\right)$, which is the total absolute curvature of the dual surface $f^{\#}$. In Part I, we investigated surfaces with low TA $\left(f^{\#}\right)$. Here we investigate CMC-1 surfaces with low TA $(f)$.

Classifying CMC-1 surfaces in $H^{3}$ with low TA $(f)$ is more difficult than classifying those with low $\mathrm{TA}\left(f^{\#}\right)$, for the following reasons: $\mathrm{TA}(f)$ equals the area of the spherical image of the (holomorphic) secondary Gauss map $g$, and $g$ might not be single-valued on the surface. Therefore, TA $(f)$ is generally not a $4 \pi$-multiple of an integer, unlike the case of $\operatorname{TA}\left(f^{\#}\right)$. Furthermore, the Osserman inequality does not hold for $\operatorname{TA}(f)$, also unlike the case of TA $\left(f^{\#}\right)$. The weaker Cohn-Vossen inequality is the best general lower bound for $\operatorname{TA}(f)$ (with equality never holding 19). In Section 3 , we shall prove the following:

Theorem 1.1. Let $f: M^{2} \rightarrow H^{3}$ be a complete CMC-1 immersion of total absolute curvature $\mathrm{TA}(f) \leq 4 \pi$. Then $f$ is either

- a horosphere,

- an Enneper cousin,

- an embedded catenoid cousin,

- a finite $\delta$-fold covering of an embedded catenoid cousin with $M^{2}=\boldsymbol{C} \backslash\{0\}$ and secondary Gauss map $g=z^{\mu}$ for $\mu \leq 1 / \delta$, or

- a warped catenoid cousin with injective secondary Gauss map.

The horosphere is the only flat (and consequently totally umbilic) CMC-1 surface in $H^{3}$. The catenoid cousins are the only CMC-1 surfaces of revolution [3]. The Enneper cousins are isometric to minimal Enneper surfaces [3]. The warped catenoid cousins [19] are less well known and are described in Section 2.

Although this theorem is simply stated, for the reasons stated above the proof is more delicate than it would be if the condition $\mathrm{TA}(f) \leq 4 \pi$ were replaced with $\mathrm{TA}\left(f^{\#}\right) \leq 4 \pi$, or if minimal surfaces in $\boldsymbol{R}^{3}$ with $\mathrm{TA} \leq 4 \pi$ were considered. CMC-1 surfaces $f$ with $\operatorname{TA}\left(f^{\#}\right) \leq 4 \pi$ are shown in Part I to be only horospheres, Enneper

Date: Feburuary 05, 2001. 
cousin duals, catenoid cousins, and warped catenoid cousins with embedded ends. It is well known that the only complete minimal surfaces in $\boldsymbol{R}^{3}$ with TA $\leq 4 \pi$ are the plane, the Enneper surface, and the catenoid.

We see from this theorem that any three-ended surface $f$ satisfies $\mathrm{TA}(f)>4 \pi$, and so the Cohn-Vossen inequality is not sharp for such $f$. On the other hand, the Cohn-Vossen inequality is sharp for catenoid cousins, and a numerical experiment in 15 shows it to be sharp for genus 0 surfaces with 4 ends. This raises the question:

Which classes of surfaces $f$ have a stronger lower bound for $\mathrm{TA}(f)$

than that given by the Cohn-Vossen inequality?

Pursuing this, in Section 1 we show that stronger lower bounds exist for genus zero CMC-1 surfaces with an odd number of ends.

We extend Theorem 1.1 in a follow-up work 15], to find an inclusive list of possibilities for CMC-1 surfaces with $\mathrm{TA}(f) \leq 8 \pi$, and we consider which possibilities we can classify or find examples for. (Minimal surfaces in $\boldsymbol{R}^{3}$ with TA $\leq 8 \pi$ are classified by Lopez [9]. Those with TA $\leq 4 \pi$ are listed in Table 1.)

\section{Preliminaries}

Let $f: M \rightarrow H^{3}$ be a conformal CMC-1 immersion of a Riemann surface $M$ into $H^{3}$. Let $d s^{2}, d A$ and $K$ denote the induced metric, induced area element and Gaussian curvature, respectively. Then $K \leq 0$ and $d \sigma^{2}:=(-K) d s^{2}$ is a conformal pseudometric of constant curvature 1 on $M$. We call the developing map $g: \widetilde{M}:=$ (the universal cover of $M) \rightarrow \boldsymbol{C P}^{1}$ the secondary Gauss map of $f$. Namely, $g$ is a conformal map so that its pull-back of the Fubini-Study metric of $\boldsymbol{C P}^{1}$ equals $d \sigma^{2}$ :

$$
d \sigma^{2}=(-K) d s^{2}=\frac{4 d g d \bar{g}}{(1+g \bar{g})^{2}} .
$$

By definition, the secondary Gauss map $g$ of the immersion $f$ is uniquely determined up to transformations of the form

$$
g \mapsto a \star g:=\frac{a_{11} g+a_{12}}{a_{21} g+a_{22}} \quad a=\left(\begin{array}{ll}
a_{11} & a_{12} \\
a_{21} & a_{22}
\end{array}\right) \in \mathrm{SU}(2) .
$$

In addition to $g$, two other holomorphic invariants $G$ and $Q$ are closely related to geometric properties of CMC-1 surfaces. The hyperbolic Gauss map $G: M \rightarrow \boldsymbol{C P}^{1}$ is holomorphic and is defined geometrically by identifying the ideal boundary of $H^{3}$ with $\boldsymbol{C P}^{1}: G(p)$ is the asymptotic class of the normal geodesic of $f(M)$ starting at $f(p)$ and oriented in the mean curvature vector's direction. The Hopf differential $Q$ is the symmetric holomorphic 2-differential on $M$ such that $-Q$ is the $(2,0)$-part of the complexified second fundamental form. The Gauss equation implies

$$
d s^{2} \cdot d \sigma^{2}=4 Q \cdot \bar{Q},
$$

where means the symmetric product. Moreover, these invariants are related by

$$
S(g)-S(G)=2 Q,
$$

where $S(\cdot)$ denotes the Schwarzian derivative

$$
S(h):=\left[\left(\frac{h^{\prime \prime}}{h^{\prime}}\right)^{\prime}-\frac{1}{2}\left(\frac{h^{\prime \prime}}{h^{\prime}}\right)^{2}\right] d z^{2} \quad\left({ }^{\prime}=\frac{d}{d z}\right)
$$

with respect to a complex coordinate $z$ on $M$. 
Since $K \leq 0$, we can define the total absolute curvature as

$$
\mathrm{TA}(f):=\int_{M}(-K) d A \in[0,+\infty] .
$$

Then TA $(f)$ is the area of the image in $\boldsymbol{C P}^{1}$ of the secondary Gauss map. TA $(f)$ is generally not an integer multiple of $4 \pi$ - for catenoid cousins [3, Example 2] and their $\delta$-fold covers, TA $(f)$ admits any positive real number.

For each conformal CMC-1 immersion $f: M \rightarrow H^{3}$, there is a holomorphic null immersion $F: \widetilde{M} \rightarrow \mathrm{SL}(2, \boldsymbol{C})$, the lift of $f$, satisfying the differential equation

$$
d F=F\left(\begin{array}{cc}
g & -g^{2} \\
1 & -g
\end{array}\right) \omega, \quad \omega=\frac{Q}{d g}
$$

such that $f=F F^{*}$, where $F^{*}={ }^{t} \bar{F}$. Here we consider $H^{3}=\operatorname{SL}(2, \boldsymbol{C}) / \mathrm{SU}(2)=$ $\left\{a a^{*} \mid a \in \mathrm{SL}(2, \boldsymbol{C})\right\}$. If $F=\left(F_{i j}\right)$, equation (2.5) implies

$$
g=-\frac{d F_{12}}{d F_{11}}=-\frac{d F_{22}}{d F_{21}},
$$

and it is shown in [3] that

$$
G=\frac{d F_{11}}{d F_{21}}=\frac{d F_{12}}{d F_{22}} .
$$

We now assume that the induced metric $d s^{2}$ on $M$ is complete and that $\operatorname{TA}(f)<$ $\infty$, hence there exists a compact Riemann surface $\bar{M}_{\gamma}$ of genus $\gamma$ and a finite set of points $\left\{p_{1}, \ldots, p_{n}\right\} \subset \bar{M}_{\gamma}(n \geq 1)$ so that $M$ is biholomorphic to $\bar{M}_{\gamma} \backslash\left\{p_{1}, \ldots, p_{n}\right\}$. We call the points $p_{j}$ the ends of $f$. Moreover, the pseudometric $d \sigma^{2}$ as in (2.1) is an element of $\operatorname{Met}_{1}\left(\bar{M}_{\gamma}\right)$ ([3, Theorem 4], for a definition of $\mathrm{Met}_{1}$ see Appendix A $)$.

Unlike the Gauss map for minimal surfaces with TA $<\infty$ in $\boldsymbol{R}^{3}$, the hyperbolic Gauss map $G$ of $f$ might not extend to a meromorphic function on $\bar{M}_{\gamma}$ (as the Enneper cousins show). However, the Hopf differential $Q$ does extend to a meromorphic differential on $\bar{M}_{\gamma}$ [3]. We say an end $p_{j}(j=1, \ldots, n)$ of a CMC-1 immersion is regular if $G$ is meromorphic at $p_{j}$. When $\operatorname{TA}(f)<\infty$, an end $p_{j}$ is regular precisely when the order of $Q$ at $p_{j}$ is at least -2 , and otherwise $G$ has an essential singularity at $p_{j}[19]$.

Thus the orders of $Q$ at the ends $p_{j}$ are important for understanding the geometry of the surface, so we now introduce a notation that reflects this. We say a CMC1 surface is of type $\boldsymbol{\Gamma}\left(d_{1}, \ldots, d_{n}\right)$ if it is given as a conformal immersion $f: \bar{M}_{\gamma} \backslash$ $\left\{p_{1}, \ldots, p_{n}\right\} \rightarrow H^{3}$, where $\operatorname{ord}_{p_{j}} Q=d_{j}$ for $j=1, \ldots, n$ (for example, if $Q=z^{-2} d z^{2}$ at $p_{1}=0$, then $d_{1}=-2$ ). We use $\boldsymbol{\Gamma}$ because it is the capitalized form of $\gamma$, the genus of $\bar{M}_{\gamma}$. For instance, $\mathbf{I}(-4)$ is the class of surfaces of genus 1 with 1 end so that $Q$ has an order 4 pole at the end, and $\mathbf{O}(-2,-3)$ is the class of surfaces of genus 0 with two ends so that $Q$ has an order 2 pole at one end and an order 3 pole at the other.

We close this section with a description of the warped catenoid cousins. Here is a slightly refined version of Theorem 6.2 in 19 :

Theorem 2.1. A complete conformal CMC-1 immersion $f: M=C \backslash\{0\} \rightarrow H^{3}$ with two regular ends is a $\delta$-fold cover of a catenoid cousin (which is characterized by $g=z^{\mu}$ and $\omega=\left(1-\mu^{2}\right) z^{-\mu-1} d z /(4 \mu)$ for $\mu \in \boldsymbol{R}$ ), or an immersion (or possibly 

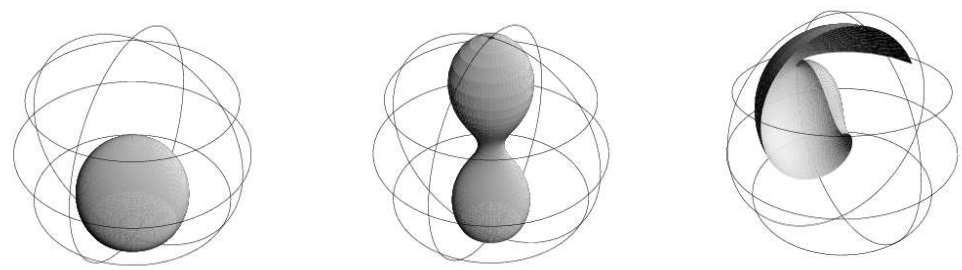

Figure 1. A horosphere, a catenoid cousin with $g=z^{\mu}(\mu=$ 0.8 ), and a fundamental piece (one-fourth of the surface with the end cut away) of an Enneper cousin with $g=z, Q=(1 / 2) d z^{2}$.
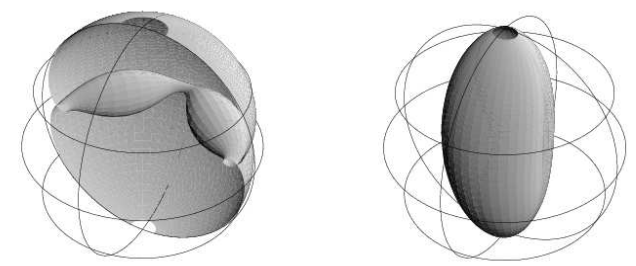

Figure 2. Two warped catenoid cousins, the first with $\delta=1$, $l=4, b=1 / 2$ and the second with $\delta=2, l=1, b=1 / 2$. (Half of the first surface has been cut away.) Only the second of these two surfaces has $\operatorname{TA}(f)=4 \pi$ (since $l=1$ ), even though its ends are not embedded.
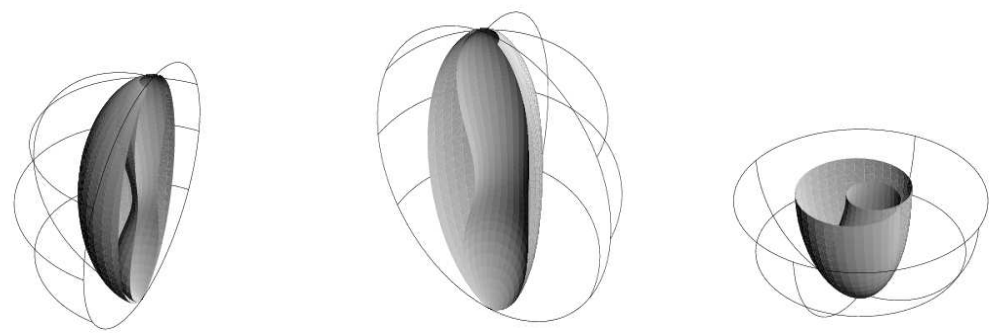

FiguRE 3. Cut-away views of the second warped catenoid cousin in Figure 2.

a finite covering of it) where $g$ and $\omega$ can be chosen as

$$
g=\frac{\delta^{2}-l^{2}}{4 l} z^{l}+b, \quad \omega=\frac{Q}{d g}=z^{-l-1} d z,
$$

with $l, \delta \in \boldsymbol{Z}^{+}, l \neq \delta$, and $b \geq 0$. 
When $b=0, f$ is a $\delta$-fold cover of a catenoid cousin with $\mu=l$. When $b>0$, we call $f$ a warped catenoid cousin, and its discrete symmetry group is the natural $\boldsymbol{Z}_{2}$ extension of the dihedral group $D_{l}$. Furthermore, the warped catenoid cousins can be written explicitly as

$$
f=F F^{*}, \quad F=F_{0} B,
$$

where

$$
F_{0}=\sqrt{\frac{\delta^{2}-l^{2}}{\delta}}\left(\begin{array}{cc}
\frac{1}{l-\delta} z^{(\delta-l) / 2} & \frac{\delta-l}{4 l} z^{(l+\delta) / 2} \\
\frac{1}{l+\delta} z^{-(l+\delta) / 2} & \frac{-(l+\delta)}{4 l} z^{(l-\delta) / 2}
\end{array}\right) \quad \text { and } \quad B=\left(\begin{array}{rr}
1 & -b \\
0 & 1
\end{array}\right) .
$$

Proof. In [19] it is shown that a complete conformal CMC-1 immersion of $M=$ $\boldsymbol{C} \backslash\{0\}$ with regular ends is a finite cover of a catenoid cousin or an immersion determined by

$$
g=a z^{l}+\hat{b}, \quad \omega=c z^{-l-1} d z,
$$

where $l$ is a nonzero integer and $a, \hat{b}$ and $c$ are complex numbers, which satisfy $l^{2}+4 a c l=\delta^{2}$ for a positive integer $\delta$ and $a, c \neq 0$. (The proof in [19] contains typographical errors: The exponents $\mu$ and $-\mu$ in equations (6.13) and (6.14) should be reversed. If $\mu \notin Z^{+}$, then the last paragraph of Case 1 is correct. If $\mu \in Z^{+}$, then one must consider a possibility that is included in Case 2 in that proof, and the result follows.) Changing $z$ to $1 / z$ if necessary, we may assume $l \geq 1$.

Choose $\theta$ so that $b:=\hat{b} e^{2 i \theta} \geq 0$. Doing the $\mathrm{SU}(2)$ transformation

$$
g \mapsto\left(\begin{array}{cc}
e^{i \theta} & 0 \\
0 & e^{-i \theta}
\end{array}\right) \star g, \quad \omega \mapsto e^{-2 i \theta} \omega,
$$

and replacing $z$ with $e^{-2 i \theta / l} c^{1 / l} z$ produces the same surface, and one has

$$
g=a c z^{l}+b, \quad \omega=z^{-l-1} d z, \quad a c=\frac{\delta^{2}-l^{2}}{4 l} .
$$

Thus $g$ and $\omega$ are as desired.

To study the symmetry group of the surface, we consider the transformations

$$
\phi_{\varrho}(z)=e^{2 \pi i \varrho / l} \bar{z} \quad(\varrho \in \boldsymbol{Z}), \quad \text { and } \quad \phi(z)=\left(\frac{16 l^{2}\left(1+b^{2}\right)}{\left(\delta^{2}-l^{2}\right)^{2}}\right)^{1 / l} \frac{1}{\bar{z}}
$$

of the plane. Then the Hopf differential and secondary Gauss map change as

$$
\overline{Q \circ \phi_{\varrho}}=Q, \quad \overline{g \circ \phi_{\varrho}}=g, \quad \overline{Q \circ \phi}=Q, \quad \overline{g \circ \phi}=\frac{b g+1}{g-b}=A \star g \text {, }
$$

where

$$
A=\frac{i}{\sqrt{1+b^{2}}}\left(\begin{array}{rr}
b & 1 \\
1 & -b
\end{array}\right) \in \mathrm{SU}(2) .
$$

Hence $\phi_{\varrho}$ and $\phi$ represent isometries of the surface. One can then check that there are no other isometries of the surface, i.e., that there are no other anti-conformal bijections $\hat{\phi}$ of $M$ so that $\overline{Q \circ \hat{\phi}}=Q$ and $\overline{g \circ \hat{\phi}}=A \star g$ for some $A \in S U(2)$. Thus the symmetry group is $D_{l} \times \boldsymbol{Z}_{2}$.

To see that the warped catenoid cousins have the explicit representation described in the theorem, one needs only to verify that $F=F_{0} B$ satisfies (2.5). 


\begin{tabular}{|l||c|l|}
\hline Type & TA & The surface \\
\hline \hline $\mathbf{O}(0)$ & 0 & Plane \\
\hline $\mathbf{O}(-4)$ & $4 \pi$ & Enneper surface \\
\hline $\mathbf{O}(-2,-2)$ & $4 \pi$ & Catenoid \\
\hline
\end{tabular}

TABLE 1. Classification of minimal surfaces in $\boldsymbol{R}^{3}$ with TA $\leq 4 \pi$.

\begin{tabular}{|l||c|l|}
\hline Type & TA $(f)$ & The surface \\
\hline \hline $\mathbf{O}(0)$ & 0 & Horosphere \\
\hline $\mathbf{O}(-4)$ & $4 \pi$ & Enneper cousins \\
\hline $\mathbf{O}(-2,-2)$ & $(0,4 \pi]$ & $\begin{array}{c}\text { Catenoid cousins and } \\
\text { their } \delta \text {-fold covers }\end{array}$ \\
\hline $\mathbf{O}(-2,-2)$ & $4 \pi$ & $\begin{array}{c}\text { Warped catenoid } \\
\text { cousins with } l=1\end{array}$ \\
\hline
\end{tabular}

TABle 2. Classification of CMC-1 surfaces in $H^{3}$ with TA $(f) \leq 4 \pi$.

\section{Complete CMC-1 surfaces with TA $(f) \leq 4 \pi$}

In this section we will prove Theorem 1.1. First we prepare some notations and basic facts. For a complete conformal CMC-1 immersion $f: M=\bar{M}_{\gamma} \backslash$ $\left\{p_{1}, \ldots, p_{n}\right\} \rightarrow H^{3}$, we define $\mu_{j}$ and $\mu_{j}^{\#}$ to be the branching orders of the Gauss maps $g$ and $G$, respectively, at each end $p_{j}$. At an irregular end $p_{j}$, we have $\mu_{j}^{\#}=\infty$. At a regular end $p_{j}$, relation (2.4) implies that the Hopf differential $Q$ expands as

$$
Q=\left(\frac{1}{2} \frac{c_{j}}{\left(z-p_{j}\right)^{2}}+\ldots\right) d z^{2}, \quad c_{j}=-\frac{1}{2} \mu_{j}\left(\mu_{j}+2\right)+\frac{1}{2} \mu_{j}^{\#}\left(\mu_{j}^{\#}+2\right),
$$

where $z$ is a local complex coordinate around $p_{j}$.

Let $\left\{q_{1}, \ldots, q_{m}\right\} \subset M$ be the $m$ umbilic points of the surface, and let $\xi_{k}=$ $\operatorname{ord}_{q_{k}} Q$. Then, as in (2.5) of Part I,

$$
\sum_{j=1}^{n} d_{j}+\sum_{k=1}^{m} \xi_{k}=4 \gamma-4, \quad \text { in particular, } \quad \sum_{j=1}^{n} d_{j} \leq 4 \gamma-4 .
$$

By (2.3) and (2.4), it holds that

$$
\xi_{k}=\left[\text { branch order of } G \text { at } q_{k}\right]=\left[\text { branch order of } g \text { at } q_{k}\right]=\operatorname{ord}_{q_{k}} d \sigma^{2} .
$$

As in (2.4) of Part I, the Gauss-Bonnet theorem implies

$$
\frac{\mathrm{TA}(f)}{2 \pi}=\chi\left(\bar{M}_{\gamma}\right)+\sum_{j=1}^{n} \mu_{j}+\sum_{k=1}^{m} \xi_{k}
$$

where $\chi$ denotes the Euler characteristic. Combining this with (3.2), we have

$$
\frac{\mathrm{TA}(f)}{2 \pi}=2 \gamma-2+\sum_{j=1}^{n}\left(\mu_{j}-d_{j}\right)
$$

Proposition 4.1 in 19 implies that

$$
\mu_{j}-d_{j}>1, \quad \text { in particular, } \quad \mu_{j}-d_{j} \geq 2 \text { if } \mu_{j} \in \boldsymbol{Z} .
$$


An end $p_{j}$ is regular if and only if $d_{j} \geq-2$, and then $G$ is meromorphic at $p_{j}$. Thus

$$
\mu_{j}^{\#} \text { is a non-negative integer if } d_{j} \geq-2 \text {. }
$$

By Proposition 4 of [3],

$$
\mu_{j}>-1
$$

hence equation (3.1) implies

$$
\mu_{j}=\mu_{j}^{\#} \in Z \quad \text { if } d_{j} \geq-1 .
$$

Finally, we note that

$$
\begin{aligned}
& \text { any meromorphic function on a Riemann surface } \bar{M}_{\gamma} \text { of genus } \\
& \gamma \geq 1 \text { has at least three distinct branch points. }
\end{aligned}
$$

To prove this, let $\varphi$ be a meromorphic function on $\bar{M}_{\gamma}$ with $N$ branch points $\left\{q_{1}, \ldots, q_{N}\right\}$ of branching order $\psi_{k}$ at $q_{k}$. Then the Riemann-Hurwicz relation implies

$$
2 \operatorname{deg} \varphi=2-2 \gamma+\sum_{k=1}^{N} \psi_{k} .
$$

On the other hand, since the multiplicity of $\varphi$ at $q_{k}$ is $\psi_{k}+1, \operatorname{deg} \varphi \geq \psi_{k}+1$ $(k=1, \ldots, N)$. Thus

$$
(N-2) \operatorname{deg} \varphi \geq 2(\gamma-1)+N .
$$

If $\gamma \geq 1$, then $\operatorname{deg} \varphi \geq 2$, and so $N \geq 3$.

Remark. Facts (3.4) and (3.5) imply that, for CMC-1 surfaces, equality never holds in the Cohn-Vossen inequality [19]:

$$
\frac{\mathrm{TA}(f)}{2 \pi}>-\chi(M)=n-2+2 \gamma .
$$

Proof of Theorem 1.1. By (3.4),

$$
2 \geq \frac{\mathrm{TA}(f)}{2 \pi}=2 \gamma-2+\sum_{j=1}^{n}\left(\mu_{j}-d_{j}\right) .
$$

Since $\mu_{j}-d_{j}>1$ by (3.5), we have

$$
4>2 \gamma+n .
$$

Thus the only possibilities are

$$
(\gamma, n)=(0,1), \quad(0,2), \quad(0,3), \quad(1,1) .
$$

The case $(\boldsymbol{\gamma}, \boldsymbol{n})=(\mathbf{1}, \mathbf{1})$. By (3.11) and (3.7), we have $d_{1} \geq \mu_{1}-2>-3$. Thus the end $p_{1}$ is regular, and $G$ is meromorphic on $\bar{M}_{1}$. By (3.2), $d_{1} \leq 0$. If $d_{1}=-2$, the end has non-vanishing flux, and the surface does not exist, by Corollary 3 of 13. If $d_{1}=0$ or -1 , by (3.2) there is at most one umbilic point. Since any branch point of $G$ is at an end or an umbilic point, (3.9) is contradicted. Hence a surface of this type does not exist.

The case $(\gamma, n)=(\mathbf{0 , 1})$. Here the surface is simply connected, so it has a canonical isometrically corresponding minimal surface in $\boldsymbol{R}^{3}$ with the same total absolute curvature. We conclude the surface is a horosphere or an Enneper cousin. 
The case $(\gamma, \boldsymbol{n})=(\mathbf{0 , 2})$. Here, by (3.2), we have $d_{1}+d_{2} \leq-4$. On the other hand, by (3.11) and (3.7), we have $d_{1}+d_{2} \geq-4+\left(\mu_{1}+\mu_{2}\right)>-6$. Thus $d_{1}+d_{2}$ is either -4 or -5 , and we now consider these two cases separately:

The case $\boldsymbol{d}_{\mathbf{1}}+\boldsymbol{d}_{\mathbf{2}}=\mathbf{- 4}$. If $d_{1}+d_{2}=-4$, then there are no umbilic points, by (3.2). If $d_{1}, d_{2} \geq-2$, then the ends are regular, and Theorem 2.1 implies the surface is a $\delta$-fold cover of an embedded catenoid cousin with $\delta \leq 1 / \mu$, or a warped catenoid cousin with $l=1$.

Now assume that

$$
d_{1} \geq-1, \quad d_{2} \leq-3 .
$$

Then we have $\mu_{1} \in \boldsymbol{Z}$ by (3.8). By Proposition A.1 in Appendix A, we cannot have just one $\mu_{j} \notin \boldsymbol{Z}$, so also $\mu_{2} \in \boldsymbol{Z}$. Then $g$ is single-valued on $M$. Since $g$ and $G$ are both single-valued on $M$, the lift $F$ is also (see equations (1.6) and (1.7) in 21]), and so the dual immersion $f^{\#}$ is also single-valued on $M$. Since $\left(f^{\#}\right)^{\#}=f, f^{\#}$ is a CMC-1 immersion with dual total absolute curvature $4 \pi$ and of type $\mathbf{O}(-1,-3)$. Such an $f^{\#}$ cannot exist by Theorem 3.1 of Part I, so such an $f$ does not exist.

The case $\boldsymbol{d}_{\mathbf{1}}+\boldsymbol{d}_{\mathbf{2}}=\mathbf{- 5}$. If $d_{1}+d_{2}=-5$, then the surface has only one umbilic point $q_{1}$ with $\xi_{1}=1$, by (3.2), and we can set $\bar{M}_{0}=C \cup\{\infty\}, p_{1}=0, p_{2}=\infty$, and $q_{1}=1$.

By (3.11), $\mu_{1}+\mu_{2} \leq-1$. Then by (3.7) at least one of $\mu_{1}$ and $\mu_{2}$ is not an integer. Hence both are not integers, by Proposition A.1 in Appendix A. Then (3.8) implies we may assume $d_{1}=-2$ and $d_{2}=-3$. by Proposition A.2 in Appendix A, the metric $d \sigma^{2}$ is the pull-back of the Fubini-Study metric on $\boldsymbol{C P}^{1}$ by the map

$$
g=c z^{\mu}\left(z-\frac{\mu+1}{\mu}\right) \quad(c \in \boldsymbol{C} \backslash\{0\}, \mu \in \boldsymbol{R} \backslash\{0, \pm 1\}) .
$$

On the other hand, the Hopf differential is of the form

$$
Q(z)=q(z) d z^{2}=\theta \frac{z-1}{z^{2}} d z^{2} \quad(\theta \in C \backslash\{0\}) .
$$

Thus $\omega=Q / d g$ can be written in the form

$$
\omega=w(z) d z=\frac{\theta}{c} \frac{1}{\mu+1} \frac{1}{z^{\mu+1}} d z .
$$

Consider the equation (which is introduced in [19] as (E.1))

$$
X^{\prime \prime}+a(z) X^{\prime}+b(z) X=0, \quad\left(a(z):=-\frac{w^{\prime}(z)}{w(z)}, \quad b(z):=-q(z)\right) .
$$

We expand the coefficients $a$ and $b$ as

$$
a(z)=\frac{1}{z} \sum_{j=0}^{\infty} a_{j} z^{j}, \quad b(z)=\frac{1}{z^{2}} \sum_{j=0}^{\infty} b_{j} z^{j},
$$

Then the origin $z=0$ is a regular singularity of equation (3.14). Let $\lambda$ and $\lambda+m$ be the solutions of the corresponding indicial equation $t(t-1)+a_{0} t+b_{0}=0$ with $m \geq 0$. If the surface exists, then Theorem 2.4 of [19] implies that $m$ must be a positive integer and the log-term coefficient of the solutions of (3.14) must vanish. When $m \in \boldsymbol{Z}^{+}$, the log-term coefficient vanishes if and only if

$$
\sum_{k=0}^{m-1}\left\{(\lambda+k) a_{m-k}+b_{m-k}\right\} \eta_{k}(\lambda)=0
$$


where $\eta_{0}=1$ and $\eta_{1}, \ldots, \eta_{m-1}$ are given recursively by

$$
\eta_{j}=\frac{1}{j(m-j)} \sum_{k=0}^{j-1}\left\{(\lambda+k) a_{j-k}+b_{j-k}\right\} \eta_{k}
$$

as in Proposition A.3 in Appendix A of Part I. Here we have

$$
0=a_{1}=a_{2}=\ldots, \quad 0=b_{2}=b_{3}=\ldots,
$$

and so the log-term coefficient never vanishes at the end $p_{1}$, because $b_{1}=-\theta \neq 0$. Thus this type of surface does not exist.

The case $(\gamma, \boldsymbol{n})=(\mathbf{0}, \mathbf{3})$. This is the only remaining case. But this type of surface does not exist, by the following Theorem 3.1.

Theorem 3.1. Let $f: M \rightarrow H^{3}$ be a complete CMC-1 immersion of genus zero with three ends. Then $\mathrm{TA}(f)>4 \pi$.

Remark. The second and third authors proved that $\mathrm{TA}(f) \geq 4 \pi$ holds for CMC-1 surfaces of genus 0 with three ends [24, Proposition 2.7]. Then the essential part of Theorem 3.1 is that $\mathrm{TA}(f)=4 \pi$ is impossible.

Proof of Theorem 3.1. We suppose TA $(f)=4 \pi$, and will arrive at a contradiction. Without loss of generality, we may set $\bar{M}_{0}=\boldsymbol{C} \cup\{\infty\}$ and $p_{1}=0, p_{2}=1$, and $p_{3}=\infty$.

Step 1. Since $\gamma=0$ and $\mathrm{TA}(f) \leq 4 \pi$, 3.4 implies

$$
4 \geq \sum_{j=1}^{3}\left(\mu_{j}-d_{j}\right) .
$$

Since $\mu_{j}-d_{j}>1$ for all $j, 3.15$ implies $\mu_{j}-d_{j}<2$ for all $j$. Hence $\mu_{1}, \mu_{2}, \mu_{3} \notin \boldsymbol{Z}$ by (3.5). Then (3.8) implies $d_{j} \leq-2$ for all $j$, and as equations (3.15) and (3.7) imply $d_{1}+d_{2}+d_{3} \geq-4+\mu_{1}+\mu_{2}+\mu_{3}>-7$, we have

$$
d_{1}=d_{2}=d_{3}=-2 \text {, }
$$

and so the ends are regular.

On the other hand, since $\mathrm{TA}(f)=4 \pi$, 3.4 and (3.16) imply

$$
\mu_{1}+\mu_{2}+\mu_{3}=-2 \text {. }
$$

Then by (3.7), we have

$$
-1<\mu_{j}<0 \quad(j=1,2,3),
$$

and furthermore at least two of the $\mu_{j}$ are less than $-1 / 2$. We may arrange the ends so that

$$
-1<\mu_{1}, \mu_{2}<-\frac{1}{2} \text { and } \quad-1<\mu_{3}<0
$$

Moreover, by Appendix A of [24] (note that the $C_{j}$ there equal $\pi\left(\mu_{j}+1\right)$ ), the metric $d \sigma^{2}$ is reducible (as defined in Appendix B of the present paper). Then, by Proposition B.1 and the relation (A.3) in the appendices here, the secondary Gauss map $g$ can be expressed in the form

$$
g=z^{-\left(\mu_{1}+1\right)}(z-1)^{\beta+1} \frac{a(z)}{b(z)},
$$


where $a(z), b(z)$ are relatively prime polynomials without zeros at $p_{1}$ and $p_{2}$, and

$$
\beta=\mu_{2} \quad \text { or } \quad \beta=-2-\mu_{2} .
$$

Note that the order of $g$ at $p_{3}=\infty$ is $\pm\left(\mu_{3}+1\right)$ and is also $\mu_{1}-\beta-\operatorname{deg} a+\operatorname{deg} b$. If $\beta=\mu_{2}$,

$$
2 \mu_{1}=\operatorname{deg} a-\operatorname{deg} b-1 \quad \text { or } \quad 2 \mu_{2}=\operatorname{deg} b-\operatorname{det} a-1
$$

holds. Thus either $2 \mu_{1}$ or $2 \mu_{2}$ is an integer, but this contradicts (3.19), so $\beta=$ $-\mu_{2}-2$ :

$$
g=z^{-\mu_{1}-1}(z-1)^{-\mu_{2}-1} \frac{a(z)}{b(z)} .
$$

Thus, by (3.17), we have

$$
-\mu_{3}-\operatorname{deg} a+\operatorname{deg} b= \pm\left(\mu_{3}+1\right) .
$$

Hence either

$$
\begin{gathered}
\operatorname{deg} a-\operatorname{deg} b=1 \quad \text { and the order of } g \text { at } \infty \text { is }-\mu_{3}-1, \text { or } \\
\mu_{3}=-1 / 2, \operatorname{deg} a=\operatorname{deg} b \quad \text { and the order of } g \text { at } \infty \text { is } \mu_{3}+1
\end{gathered}
$$

holds because of (3.19). To get more specific information about $a(z)$ and $b(z)$, we now consider $d g$ :

Step 2. Since $Q$ is holomorphic on $C \backslash\{0,1\}$ with two zeroes (by (3.2)), (3.1) implies

$$
Q=\frac{1}{2}\left(\frac{c_{3} z^{2}+\left(c_{2}-c_{1}-c_{3}\right) z+c_{1}}{z^{2}(z-1)^{2}}\right) d z^{2},
$$

with the $c_{j}$ as in (3.1), as pointed out in [24, page 84]. Note that

$$
c_{j}>0 \quad(j=1,2,3),
$$

because $\mu_{j}^{\#} \geq 0$ and $-1<\mu_{j}<0$. Let $q_{1}$ and $q_{2}$ be the two roots of

$$
c_{3} z^{2}+\left(c_{2}-c_{1}-c_{3}\right) z+c_{1}=0 .
$$

In the case of a double root, we write $q:=q_{1}=q_{2}$.

Using (3.3) and Proposition B.1 in Appendix B, $d g$ has only the four following possibilities:

$$
\begin{aligned}
& d g=C \frac{z^{-\mu_{1}-2}(z-1)^{-\mu_{2}-2}\left(z-q_{1}\right)\left(z-q_{2}\right)}{\prod_{k=1}^{r}\left(z-a_{k}\right)^{2}} d z, \\
& d g=C \frac{z^{-\mu_{1}-2}(z-1)^{-\mu_{2}-2}\left(z-q_{1}\right)}{\left(z-q_{2}\right)^{3} \prod_{k=1}^{r}\left(z-a_{k}\right)^{2}} d z \quad\left(q_{1} \neq q_{2}\right), \\
& d g=C \frac{z^{-\mu_{1}-2}(z-1)^{-\mu_{2}-2}}{\left(z-q_{1}\right)^{3}\left(z-q_{2}\right)^{3} \prod_{k=1}^{r}\left(z-a_{k}\right)^{2}} d z \quad\left(q_{1} \neq q_{2}\right),
\end{aligned}
$$

or

$$
d g=C \frac{z^{-\mu_{1}-2}(z-1)^{-\mu_{2}-2}}{(z-q)^{4} \prod_{k=1}^{r}\left(z-a_{k}\right)^{2}} d z \quad\left(q=q_{1}=q_{2}\right)
$$


where $r$ is a non-negative integer and the points $a_{k} \in C \backslash\left\{0,1, q_{1}, q_{2}\right\}$ are mutually distinct. In the first case (3.28), the order of $d g$ at infinity $\left(z=p_{3}=\infty\right)$ is given by

$$
\mu_{1}+\mu_{2}+2 r=2 r-2-\mu_{3}=\mu_{3} \text { or }-\mu_{3}-2 .
$$

So $2 r-2=2 \mu_{3} \in(-2,0)$ or $2 r-2-\mu_{3}=-\mu_{3}-2$. Hence $r=0$ and the order of $d g$ at $\infty$ is $-\mu_{3}-2$ in the first case.

In the other three cases (3.29), (3.30), (3.31), the orders of $d g$ at infinity are

$$
\mu_{1}+\mu_{2}+(2 \text { or } 6 \text { or } 4)+2 r+2 \geq 2-\mu_{3}+2 r>2,
$$

respectively. These orders must equal either $\mu_{3}<0$ or $-\mu_{3}-2<0$, so none of these three cases can occur. We conclude that $d g$ is of the form

$$
d g=C z^{-\mu_{1}-2}(z-1)^{-\mu_{2}-2}\left(z-q_{1}\right)\left(z-q_{2}\right) d z \quad(C \in C \backslash\{0\}) .
$$

Since the order of $d g$ at $\infty$ is $\mu_{1}+\mu_{2}=-\mu_{3}-2<0$, (3.23) holds.

Step 3. Now we determine the polynomials $a(z), b(z)$ in expression (3.22). Differentiating (3.22), we have

$$
d g=\frac{z^{-\mu_{1}-2}(z-1)^{-\mu_{2}-2}}{b^{2}(z)} f(z) d z
$$

where

$$
f(z)=-\left(1+\mu_{1}\right)(z-1) a b-\left(1+\mu_{2}\right) z a b+z(z-1)\left(a^{\prime} b-a b^{\prime}\right) .
$$

Since $a(z)$ and $b(z)$ are relatively prime, $b(z)$ does not divide $f(z)$ when $\operatorname{deg} b \geq 1$. But (3.32) and (3.33) imply that $b^{2}(z)$ divides $f(z)$, so $b(z)$ is constant, and we may assume $b=1$. Here, as seen in the previous step, (3.23) holds, and then, $\operatorname{deg} a=1$. Thus we have

$$
a(z)=a_{1} z+a_{0} \quad \text { and } \quad b=1 \quad\left(a_{1} \neq 0\right) .
$$

Step 4: By (3.32), (3.33), (3.34) and (3.35) we have

$$
\begin{aligned}
-a_{1}\left(\mu_{1}+\mu_{2}+1\right) z^{2}+\left\{\mu_{1} a_{1}-\left(\mu_{1}+\mu_{2}+2\right) a_{0}\right\} z & +\left(1+\mu_{1}\right) a_{0} \\
& =C\left(z-q_{1}\right)\left(z-q_{2}\right) .
\end{aligned}
$$

Equation (3.27) also has roots $q_{1}$ and $q_{2}$, so

$$
q_{1} q_{2}=\frac{a_{0}}{a_{1}} \frac{1+\mu_{1}}{1+\mu_{3}}=\frac{c_{1}}{c_{3}}, \quad q_{1}+q_{2}=-\frac{\mu_{3} a_{0}+\mu_{1} a_{1}}{a_{1}\left(1+\mu_{3}\right)}=\frac{c_{1}}{c_{3}}+1-\frac{c_{2}}{c_{3}} .
$$

By (3.7), (3.26) and the first equation of (3.37), we have $a_{0} / a_{1}>0$. Substituting the first equation of (3.37) into the second, we have

$$
\frac{c_{2}}{c_{3}}=-\frac{1+\mu_{2}}{1+\mu_{3}}\left(\frac{a_{0}}{a_{1}}+1\right) .
$$

Since $a_{0} / a_{1}>0$, (3.7) implies $c_{2} / c_{3}<0$, contradicting (3.26) and completing the proof. 


\section{Improvement of the Cohn-Vossen Inequality}

For a complete CMC-1 immersion $f$ into $H^{3}$, equality in the Cohn-Vossen inequality never holds ([19, Theorem 4.3]). In particular, when $f$ is of genus 0 with $n$ ends,

$$
\mathrm{TA}(f)>2 \pi(n-2) \text {. }
$$

For $n=2$, the catenoid cousins show that 4.1) is sharp. But Theorem 3.1 implies

$$
\mathrm{TA}(f)>4 \pi \quad \text { for } \quad n=3,
$$

which is stronger than the Cohn-Vossen inequality (4.1). The following theorem gives a sharper inequality than that of Cohn-Vossen when $n$ is any odd integer:

Theorem 4.1. Let $f: \boldsymbol{C} \cup\{\infty\} \backslash\left\{p_{1}, \ldots, p_{2 l+1}\right\} \rightarrow H^{3}$ be a complete conformal genus 0 CMC-1 immersion with $2 l+1$ ends, $l \in \boldsymbol{Z}$. Then

$$
\mathrm{TA}(f) \geq 4 \pi l .
$$

To prove this, we first prepare two lemmas and a proposition.

Lemma 4.2. Let $\theta_{1}, \theta_{2}, \theta_{3} \in[0, \pi]$ be three real numbers such that

$$
\cos ^{2} \theta_{1}+\cos ^{2} \theta_{2}+\cos ^{2} \theta_{3}+2 \cos \theta_{1} \cos \theta_{2} \cos \theta_{3} \leq 1 .
$$

Then the following inequalities hold:

$$
\begin{aligned}
\theta_{1}+\theta_{2}+\theta_{3} & \geq \pi, \\
\theta_{2}-\theta_{1} & \leq \pi-\theta_{3} .
\end{aligned}
$$

Remark. It is well known that the inequality

$$
\cos ^{2} \theta_{1}+\cos ^{2} \theta_{2}+\cos ^{2} \theta_{3}+2 \cos \theta_{1} \cos \theta_{2} \cos \theta_{3}<1
$$

is a necessary and sufficient condition for the existence of a spherical triangle $\mathcal{T}$ with angles $\theta_{1}, \theta_{2}$ and $\theta_{3}$. Then (4.3) follows directly from the Gauss-Bonnet formula, and (4.4) is the triangle inequality for the polar triangle of $\mathcal{T}$, and the lemma follows. ( $\mathcal{T}$ 's polar triangle is the one whose vertices are the centers of the great circles containing the edges of $\mathcal{T}$.) However, we give an alternative proof:

Proof of Lemma 4.2. We set

$$
E:=\cos ^{2} \theta_{1}+\cos ^{2} \theta_{2}+\cos ^{2} \theta_{3}+2 \cos \theta_{1} \cos \theta_{2} \cos \theta_{3}-1 \leq 0 .
$$

Then

$$
\begin{aligned}
& E=4 \cos \left(\frac{\theta_{1}+\theta_{2}+\theta_{3}}{2}\right) \cos \left(\frac{-\theta_{1}+\theta_{2}+\theta_{3}}{2}\right) \times \\
& \cos \left(\frac{\theta_{1}-\theta_{2}+\theta_{3}}{2}\right) \cos \left(\frac{\theta_{1}+\theta_{2}-\theta_{3}}{2}\right) .
\end{aligned}
$$

If $\theta_{1}+\theta_{2}+\theta_{3}<\pi$, we have $\left| \pm \theta_{1} \pm \theta_{2} \pm \theta_{3}\right|<\pi$, and so

$$
\cos \left(\frac{ \pm \theta_{1} \pm \theta_{2} \pm \theta_{3}}{2}\right)>0
$$

implying $E>0$, a contradiction. This proves 4.3 . Now, since

$$
E=\cos ^{2} \theta_{1}+\cos ^{2}\left(\pi-\theta_{2}\right)+\cos ^{2}\left(\pi-\theta_{3}\right)+2 \cos \theta_{1} \cos \left(\pi-\theta_{2}\right) \cos \left(\pi-\theta_{3}\right)-1
$$

and $E \leq 0$ and $\theta_{1}, \pi-\theta_{2}, \pi-\theta_{3} \in[0, \pi]$, (4.3) implies

$$
\theta_{1}+\left(\pi-\theta_{2}\right)+\left(\pi-\theta_{3}\right) \geq \pi
$$


that is, (4.4) holds.

For a matrix $a \in \mathrm{SU}(2)$, there is a unique $C \in[0, \pi]$ so that $a$ has eigenvalues $\left\{-e^{ \pm i C}\right\}$. We define the rotation angle of $a$ as

$$
\theta(a):=2 C .
$$

In fact, if one considers the matrix acting on the unit sphere as an isometry (Möbius action on $\boldsymbol{C P}^{1}$ with the Fubini-Study metric), $\theta(a)$ is exactly the angle of rotation.

Lemma 4.3. Let $a_{0}, a_{1}, a_{2}, a_{3}$ be four matrices in $\mathrm{SU}(2)$ satisfying $a_{1} a_{2} a_{3}=a_{0}$. Then it holds that

$$
\theta\left(a_{1}\right)+\theta\left(a_{2}\right)+\theta\left(a_{3}\right) \geq \theta\left(a_{0}\right) .
$$

Proof. Setting $b:=a_{3}\left(a_{0}\right)^{-1}=\left(a_{1} a_{2}\right)^{-1}$, we have $a_{1} a_{2} b=$ id. Then Appendix A of 24 implies

$$
\cos ^{2} \frac{\theta\left(a_{1}\right)}{2}+\cos ^{2} \frac{\theta\left(a_{2}\right)}{2}+\cos ^{2} \frac{\theta(b)}{2}+2 \cos \frac{\theta\left(a_{1}\right)}{2} \cos \frac{\theta\left(a_{2}\right)}{2} \cos \frac{\theta(b)}{2} \leq 1 .
$$

So by Lemma 4.2 we have

$$
\frac{\theta\left(a_{1}\right)}{2}+\frac{\theta\left(a_{2}\right)}{2}+\frac{\theta(b)}{2} \geq \pi
$$

On the other hand, we have $a_{3}^{-1} b a_{0}=\mathrm{id}$. Again Appendix A of 24 implies

$$
\cos ^{2} \frac{\theta\left(a_{0}\right)}{2}+\cos ^{2} \frac{\theta\left(a_{3}\right)}{2}+\cos ^{2} \frac{\theta(b)}{2}+2 \cos \frac{\theta\left(a_{0}\right)}{2} \cos \frac{\theta\left(a_{3}\right)}{2} \cos \frac{\theta(b)}{2} \leq 1,
$$

since $\theta\left(a_{3}^{-1}\right)=\theta\left(a_{3}\right)$. By 4.4$)$ of Lemma 4.2, we have

$$
\frac{\theta\left(a_{0}\right)}{2}-\frac{\theta\left(a_{3}\right)}{2} \leq \pi-\frac{\theta(b)}{2}
$$

By (4.5) and (4.6), we get the assertion.

Proposition 4.4. Let $a_{1}, \ldots, a_{2 m+1}$ be matrices in $\mathrm{SU}(2)$ satisfying

$$
a_{1} a_{2} \cdots a_{2 m+1}=\mathrm{id} \text {. }
$$

Then it holds that

$$
\sum_{j=1}^{2 m+1} \theta\left(a_{j}\right) \geq 2 \pi
$$

Remark. This result does not hold for an even number of matrices: Suppose $a_{1}$, $\ldots, a_{2 m} \in \mathrm{SU}(2)$ satisfy $a_{1} a_{2} \cdots a_{2 m}=\mathrm{id}$. Then the inequality $\sum_{j=1}^{2 m} \theta\left(a_{j}\right) \geq 0$ is sharp. In fact, equality will hold if all $a_{j}=-\mathrm{id}$.

Proof of Proposition 4.4. We argue by induction. If $m=1$, the result follows from Lemma 4.3 with $a_{0}=$ id. Now suppose the result always holds for $m-1(\geq 1)$. Set

$$
b:=a_{1} a_{2} a_{3} .
$$

Then, by Lemma 4.3 .

$$
\theta\left(a_{1}\right)+\theta\left(a_{2}\right)+\theta\left(a_{3}\right) \geq \theta(b)
$$


On the other hand, we have $b a_{4} \cdots a_{2 m+1}=\mathrm{id}$, so by the inductive assumption,

$$
\theta(b)+\sum_{j=4}^{2 m+1} \theta\left(a_{j}\right) \geq 2 \pi .
$$

By (4.7) and (4.8), we get the assertion.

We now apply Proposition 4.4 to the monodromy representation of pseudometrics in $\operatorname{Met}_{1}(\boldsymbol{C} \cup\{\infty\})$ (see Appendices $\mathrm{A}$ and $\mathrm{B}$ ):

Corollary 4.5. Let $d \sigma^{2} \in \operatorname{Met}_{1}(\boldsymbol{C} \cup\{\infty\})$ with divisor

$$
D=\sum_{j=1}^{s} \beta_{j} p_{j}+\sum_{k=1}^{n} \xi_{k} q_{k}, \quad \beta_{j}>-1, \quad \xi_{k} \in \boldsymbol{Z}^{+}
$$

where the $p_{1}, \ldots, p_{s}, q_{1}, \ldots q_{n}$ are mutually distinct points in $\boldsymbol{C} \cup\{\infty\}$.

If $s+\xi_{1}+\cdots+\xi_{n}$ is an odd integer, then $\beta_{1}+\cdots+\beta_{s} \geq 1-s$.

Proof. Let $g$ be a developing map of $d \sigma^{2}$ with the monodromy representation $\rho_{g}: \pi_{1}(M) \rightarrow \mathrm{PSU}(2)=\mathrm{SU}(2) /\{ \pm \mathrm{id}\}$ on $M=\boldsymbol{C} \cup\{\infty\} \backslash\left\{p_{1}, \ldots, p_{s}, q_{1}, \ldots, q_{n}\right\}$.

$\rho_{g}$ can be lifted to an $\mathrm{SU}(2)$ representation $\tilde{\rho}_{g}: \pi_{1}(M) \rightarrow \mathrm{SU}(2)$ so that the two following properties hold:

(1) Let $T_{j}(j=1, \ldots, s)$ and $S_{k}(k=1, \ldots, n)$ be deck transformations on $\widetilde{M}$ corresponding to loops about $p_{j}$ and $q_{k}$, respectively. Then it holds that

$$
\tilde{\rho}_{g}\left(T_{1}\right) \ldots \tilde{\rho}_{g}\left(T_{s}\right) \tilde{\rho}_{g}\left(S_{1}\right) \ldots \tilde{\rho}_{g}\left(S_{n}\right)=\mathrm{id} .
$$

(2) The eigenvalues of the matrix $\tilde{\rho}_{g}\left(T_{j}\right)$ (resp. $\left.\tilde{\rho}_{g}\left(S_{k}\right)\right)$ are $\left\{-e^{ \pm i \pi\left(\beta_{j}+1\right)}\right\}$ (resp. $\left.\left\{-e^{ \pm i \pi\left(\xi_{k}+1\right)}\right\}\right)$.

This is proven in [24, Lemma 2.2] for $s=3, n=0$, and the same argument will work for general $s$ and $n$. We include an outline of the argument here: one chooses a solution $\tilde{F}$ to equation (2.12) in [24] (with $G=z$ and $Q=S(g) / 2$ ), then $\tilde{F}$ has a monodromy representation $\rho_{\tilde{F}}: \pi_{1}(M) \rightarrow \mathrm{SU}(2)$, where $\tilde{F} \rightarrow \tilde{F} \cdot \rho_{\tilde{F}}(\gamma)$ about loops $\gamma \in \pi_{1}(M)$. Then $\rho_{g}= \pm \rho_{\tilde{F}}$, and we simply choose the lift $\tilde{\rho}_{g}$ so that $\tilde{\rho}_{g}=+\rho_{\tilde{F}}$. The first property is then clear.

To show the second property, we note that when $\beta_{j}$ and $\xi_{k}$ are all given the value 0 , then $Q$ is identically 0 and so $\tilde{F}$ is constant and all $\rho_{\tilde{F}}=+$ id. Hence the eigenvalues $\left\{ \pm e^{ \pm i \pi\left(\beta_{j}+1\right)}\right\}$ (resp. $\left\{ \pm e^{ \pm i \pi\left(\xi_{k}+1\right)}\right\}$ ) of $\tilde{\rho}_{g}\left(T_{j}\right)$ (resp. $\tilde{\rho}_{g}\left(S_{k}\right)$ ) are $\left\{-e^{ \pm i \pi\left(\beta_{j}+1\right)}\right\}$ (resp. $\left\{-e^{ \pm i \pi\left(\xi_{k}+1\right)}\right\}$ ) in this case. Then, as $\beta_{j}$ and $\xi_{k}$ are deformed back to their original values, the matrices $\tilde{\rho}_{g}\left(T_{j}\right)$ (resp. $\tilde{\rho}_{g}\left(S_{k}\right)$ ) change analytically and so the sign of the eigenvalues cannot change, showing the second property.

We have

$$
\theta\left(\tilde{\rho}_{g}\left(T_{j}\right)\right) \leq 2 \pi\left(\beta_{j}+1\right)
$$

and since $\xi_{k}$ is an integer, we have

$$
\tilde{\rho}_{g}\left(S_{k}\right)=(-1)^{\xi_{k}} \text { id } .
$$

Assume $s=2 m+1$ is an odd number. Then, by the assumption, $\xi_{1}+\cdots+\xi_{n}$ is an even integer, and by (4.9) above we have $\tilde{\rho}_{g}\left(T_{1}\right) \ldots \tilde{\rho}_{g}\left(T_{2 m+1}\right)=\mathrm{id}$, so by Proposition 4.4,

$$
2 \pi \sum_{j=1}^{2 m+1}\left(\beta_{j}+1\right) \geq \sum_{j=1}^{2 m+1} \theta\left(\tilde{\rho}_{g}\left(T_{j}\right)\right) \geq 2 \pi
$$


implying the corollary when $s$ is odd.

Now suppose that $s=2 m$ is even. We have $\tilde{\rho}_{g}\left(S_{1}\right) \ldots \tilde{\rho}_{g}\left(S_{n}\right)=-\mathrm{id}$, because $\xi_{1}+\cdots+\xi_{n}$ is odd. Hence $\tilde{\rho}_{g}\left(T_{1}\right) \ldots \tilde{\rho}_{g}\left(T_{2 m}\right)(-\mathrm{id})=\mathrm{id}$, and since $\theta(-\mathrm{id})=0$, Proposition 4.4 implies

$$
2 \pi \sum_{j=1}^{2 m}\left(\beta_{j}+1\right) \geq \sum_{j=1}^{2 m} \theta\left(\tilde{\rho}_{g}\left(T_{j}\right)\right)+\theta(-\mathrm{id}) \geq 2 \pi
$$

implying the corollary when $s$ is even.

Proof of Theorem 4.1. Suppose that $\mu_{1} \in Z$. Then by (3.4) and (3.5),

$$
\frac{\mathrm{TA}(f)}{2 \pi} \geq-2+\left(\mu_{1}-d_{1}\right)+\sum_{j=2}^{2 m+1}\left(\mu_{j}-d_{j}\right)>-2+2+2 m=2 m,
$$

proving the theorem when $\mu_{1} \in \boldsymbol{Z}$.

Next, suppose that $d_{1} \leq-3$. In this case, $\mu_{1}-d_{1}>-1+3=2$. Hence again by (3.4) and (3.5), we have (4.10), and the theorem follows.

Thus we may assume $\mu_{j} \notin \boldsymbol{Z}$ and $d_{j} \geq-2$ at all ends. Then by (3.8) we have all $d_{j}=-2$. So, by (3.2) and (3.3), the corresponding pseudometric $d \sigma^{2}$ has divisor

$$
\sum_{j=1}^{2 m+1} \mu_{j} p_{j}+\sum_{k=1}^{l} \xi_{k} q_{k}, \quad \sum_{k=1}^{l} \xi_{k}=4 m-2 \in 2 \boldsymbol{Z},
$$

where $\xi_{k}=\operatorname{ord}_{q_{k}} Q$ at each umbilic point $q_{k}(k=1, \ldots, l)$. Then by Corollary 4.5 ,

$$
\mu_{1}+\mu_{2}+\cdots+\mu_{2 m+1} \geq-2 m,
$$

and so (3.4) implies the theorem.

Remark. When $m=1$, we know the lower bound $4 \pi m$ in Theorem 4.1 is sharp. However, we do not know if it is sharp for general $m$. For genus 0 CMC-1 surfaces with an even number $n \geq 4$ of ends, we do not know if there exists any stronger lower bound than that of the Cohn-Vossen inequality.

In [15], it is shown numerically that there exist CMC- 1 surfaces of genus 0 with four ends whose total absolute curvature gets arbitrarily close to $4 \pi$.

\section{Appendix A.}

For a compact Riemann surface $\bar{M}$ and points $p_{1}, \ldots, p_{n} \in \bar{M}$, a conformal metric $d \sigma^{2}$ of constant curvature 1 on $M:=\bar{M} \backslash\left\{p_{1}, \ldots, p_{n}\right\}$ is an element of $\operatorname{Met}_{1}(\bar{M})$ if there exist real numbers $\beta_{1}, \ldots, \beta_{n}>-1$ so that each $p_{j}$ is a conical singularity of order $\beta_{j}$, that is, if $d \sigma^{2}$ is asymptotic to $c_{j}\left|z-p_{j}\right|^{2 \beta_{j}} d z \cdot d \bar{z}$ at $p_{j}$, for $c_{j} \neq 0$ and $z$ a local complex coordinate around $p_{j}$. We call the formal sum

$$
D:=\sum_{j=1}^{n} \beta_{j} p_{j}
$$

the divisor corresponding to $d \sigma^{2}$. For a pseudometric $d \sigma^{2} \in \operatorname{Met}_{1}(\bar{M})$ with divisor $D$, there is a holomorphic map $g: \widetilde{M} \rightarrow \boldsymbol{C P}^{1}$ such that $d \sigma^{2}$ is the pull-back of the Fubini-Study metric of $\boldsymbol{C P}^{1}$. This map, called the developing map of $d \sigma^{2}$, is uniquely determined up to Möbius transformations $g \mapsto a \star g$ for $a \in \mathrm{SU}(2)$. 
For a conical singularity $p_{j}$ of $d \sigma^{2}$, there exists a developing map $g$ and a local coordinate $z$ of $\bar{M}$ around $p_{j}$ such that

$$
g(z)=\left(z-p_{j}\right)^{\tau_{j}} \hat{g}(z) \quad\left(\tau_{j} \in \boldsymbol{R} \backslash\{0\}\right),
$$

where $\hat{g}(z)$ is holomorphic in a neighborhood of $p_{j}$ and $\hat{g}\left(p_{j}\right) \neq 0$. Here, the order $\beta_{j}$ of $d \sigma^{2}$ at $p_{j}$ is

$$
\beta_{j}=\left\{\begin{aligned}
\tau_{j}-1 & \text { if } \tau_{j}>0, \\
-\tau_{j}-1 & \text { if } \tau_{j}<0 .
\end{aligned}\right.
$$

In other words, if $d g=\left(z-p_{j}\right)^{\beta} \hat{h}(z) d z$, where $\hat{h}(z)$ is holomorphic near $p_{j}$ and $\hat{h}\left(p_{j}\right) \neq 0$, then the order $\beta_{j}$ is expressed as

$$
\beta_{j}=\left\{\begin{array}{cl}
\beta & \text { if } \beta>-1 \\
-\beta-2 & \text { if } \beta<-1 .
\end{array}\right.
$$

The following proposition gives an obstruction to the existence of certain pseudometrics in $\operatorname{Met}_{1}(\boldsymbol{C} \cup\{\infty\})$.

Proposition A.1. For any non-integer $\beta>-1$, there is no pseudometric $d \sigma^{2}$ in $\operatorname{Met}_{1}(\boldsymbol{C} \cup\{\infty\})$ with the divisor

$$
\beta p_{1}+\sum_{j=2}^{n} m_{j} p_{j} \quad\left(m_{2}, \ldots, m_{n} \in \boldsymbol{Z}\right),
$$

where $p_{1}, \ldots, p_{n}$ are mutually distinct points in $\boldsymbol{C} \cup\{\infty\}$.

When $n=1$ (i.e. when $\sum_{j=2}^{n} m_{j} p_{j}$ is removed), this nonexistence of a "teardrop" has been pointed out in [17] and [1].

Proof. We may set $p_{1}=\infty$. Since the $m_{j} \in \boldsymbol{Z}$, the developing map $g$ of $d \sigma^{2}$ is well-defined on $\boldsymbol{C}$, and so $g$ is meromorphic on $\boldsymbol{C}$. As $d \sigma^{2}$ has finite total curvature, $g$ extends to $z=\infty$ as a holomorphic mapping. In particular, $\beta \in \boldsymbol{Z}$.

Remark. When a Riemann surface $\bar{M}_{\gamma}$ has genus $\gamma>0$, there is a pseudometric in $\operatorname{Met}_{1}\left(\bar{M}_{\gamma}\right)$ with only one singularity that has order less than 0 , by 18$]$.

Proposition A.2. Suppose a pseudometric $d \sigma^{2}$ in $\operatorname{Met}_{1}(\boldsymbol{C} \cup\{\infty\})$ has divisor

$$
\beta_{1} p_{1}+\beta_{2} p_{2}+p_{3} \quad\left(\beta_{1}, \beta_{2}>-1 \text { and } \beta_{1}, \beta_{2} \notin \boldsymbol{Z}\right),
$$

where $p_{1}:=0, p_{2}:=\infty$, and $p_{3}:=1$. Then $d \sigma^{2}$ has a developing map $g$ of the form

$$
g=c z^{\mu}\left(z-\frac{\mu+1}{\mu}\right) \quad(c \in \boldsymbol{C}, \mu \in \boldsymbol{R}),
$$

where $\beta_{1}=|\mu|-1$ and $\beta_{2}=|\mu+1|-1$.

Proof. Since $d \sigma^{2}$ has only two non-integral conical singularities, it is reducible, and Proposition B.1 in Appendix B shows that the map $g$ is written in the form

$$
g=z^{\mu} \frac{a(z)}{b(z)} \quad(\mu \notin \boldsymbol{Z}),
$$

where $a(z)$ and $b(z)$ are relatively prime polynomials with $a(0) \neq 0$ and $b(0) \neq 0$. Note that $b(z)$ can have a multiple root only at a conical singularity of $d \sigma^{2}$, hence only at $z=1$. Thus $b^{\prime}\left(z_{0}\right) \neq 0$ for all roots $z_{0} \in \boldsymbol{C} \backslash\{0,1\}$ of $b$. 
Since the change $g \mapsto 1 / g$ preserves $d \sigma^{2}$, we may assume that $\operatorname{deg} a \geq \operatorname{deg} b$. By a direct calculation, we have

$$
d g(z)=\frac{z^{\mu-1}}{b(z)^{2}} h(z) d z, \text { with } h(z)=\mu a(z) b(z)+z a^{\prime}(z) b(z)-z a(z) b^{\prime}(z) .
$$

Note that $h(0)=\mu a(0) b(0) \neq 0$.

Let $z_{0} \in \boldsymbol{C} \backslash\{0,1\}$. If $b\left(z_{0}\right) \neq 0$, then $g\left(z_{0}\right) \neq \infty$, and since $z_{0}$ is not a singularity of $d \sigma^{2}$, we have $d g\left(z_{0}\right) \neq 0$, and hence $h\left(z_{0}\right) \neq 0$. If $b\left(z_{0}\right)=0$, then $a\left(z_{0}\right) \neq 0$ and $b^{\prime}\left(z_{0}\right) \neq 0$, so $h\left(z_{0}\right) \neq 0$. Hence the only root of the polynomial $h(z)$ is 1 :

$$
h(z)=k(z-1)^{m}, \quad m \in \boldsymbol{Z}^{+}, \quad k \in \boldsymbol{C} \backslash\{0\} .
$$

We claim that $m=1$. If $b(1) \neq 0$, then $g$ (or $d \sigma^{2}$ ) having order 1 at $p_{3}=1$ means that $m=1$, by (A.3) and the above form of $d g(z)$. Suppose $b(1)=0$. Then we have $b(z)=(z-1)^{l} \hat{b}(z)$, where $\hat{b}(z)$ is a polynomial in $z$ with $\hat{b}(1) \neq 0$ and $l \in \boldsymbol{Z}^{+}$. Furthermore, $h(z)=(z-1)^{l-1} \hat{h}(z)$, where $\hat{h}(z)$ is a polynomial with $\hat{h}(1) \neq 0$, since $a(1) \neq 0$. So $m=l-1$. Then by (A.3), we have $m=1$.

Suppose that $\operatorname{deg} b \geq 1$. Since $\operatorname{deg} a \geq \operatorname{deg} b$, the top term of $h(z)$ must vanish. Thus we have $\mu=\operatorname{deg} b-\operatorname{deg} a \in \boldsymbol{Z}$, contradicting that $\beta_{1}, \beta_{2} \notin \boldsymbol{Z}$. So $b(z)$ is constant. Similarly, if $\operatorname{deg} a \geq 2$, then $\mu=-\operatorname{deg} a \in \boldsymbol{Z}$. Hence $\operatorname{deg} a=1$, and $g$ is as in A.4. $\beta_{1}=|\mu|-1$ and $\beta_{2}=|\mu+1|-1$ follow from (A.3).

\section{Appendix B.}

Consider $d \sigma^{2} \in \operatorname{Met}_{1}(\bar{M})$ with divisor $D$ as in (A.1) in Appendix A and developing map $g$. Since the Fubini-Study metric of $\boldsymbol{C P}^{1}$ is invariant under the deck transformation group $\pi_{1}(M)$ of $M:=\bar{M} \backslash\left\{p_{1}, \ldots, p_{n}\right\}$, there is a representation

$$
\rho_{g}: \pi_{1}(M) \longrightarrow \mathrm{SU}(2)
$$

such that

$$
g \circ T^{-1}=\rho_{g}(T) \star g \quad\left(T \in \pi_{1}(M)\right) .
$$

The metric $d \sigma^{2}$ is called reducible if the image of $\rho_{g}$ is a commutative subgroup in $\mathrm{SU}(2)$, and is called irreducible otherwise. Since the maximal abelian subgroup of $\mathrm{SU}(2)$ is $\mathrm{U}(1)$, the image of $\rho_{g}$ for a reducible $d \sigma^{2}$ lies in a subgroup conjugate to $\mathrm{U}(1)$, and this image might be simply the identity. We call a reducible metric $d \sigma^{2} \mathcal{H}^{3}$-reducible if the image of $\rho_{g}$ is the identity, and $\mathcal{H}^{1}$-reducible otherwise (for more on this, see [12, Section 3]).

Let $p_{1}, \ldots, p_{n-1}$ be distinct points in $\boldsymbol{C}$ and $p_{n}=\infty$. We set

$$
M_{p_{1}, \ldots, p_{n}}:=\boldsymbol{C} \cup\{\infty\} \backslash\left\{p_{1}, p_{2}, \ldots, p_{n}\right\} \quad\left(p_{n}=\infty\right)
$$

and $\widetilde{M}_{p_{1}, \ldots, p_{n}}$ its universal cover.

The following assertion was needed in the proof of Theorem 1.1 .

Proposition B.1. Let $p_{1}, \ldots, p_{n-1}$ be mutually distinct points of $\boldsymbol{C}$, and let $d \sigma^{2}$ be a metric of constant curvature 1 defined on $M_{p_{1}, \ldots, p_{n}}\left(p_{n}=\infty\right)$ which has a conical singularity at each $p_{j}$. Suppose $d \sigma^{2}$ is reducible and $\beta_{j}:=\operatorname{ord}_{p_{j}} d \sigma^{2}$ satisfy

$$
\beta_{1}, \ldots, \beta_{m} \notin \boldsymbol{Z}, \quad \beta_{m+1}, \ldots, \beta_{n-1} \in \boldsymbol{Z}, \quad \beta_{n} \notin \boldsymbol{Z},
$$

for some $m \leq n-1$. Then the metric d $\sigma^{2}$ has a developing map $g: \widetilde{M}_{p_{1}, \ldots, p_{n}} \rightarrow$ $C \cup\{\infty\}$ given by

$$
g=\left(z-p_{1}\right)^{\tau_{1}} \cdots\left(z-p_{m}\right)^{\tau_{m}} r(z) \quad\left(\tau_{1}, \ldots, \tau_{m} \in \boldsymbol{R} \backslash \boldsymbol{Z}\right),
$$


where $r(z)$ is a rational function on $\boldsymbol{C} \cup\{\infty\}$ and

$$
\left(z-p_{1}\right)^{\tau_{1}} \cdots\left(z-p_{m}\right)^{\tau_{m}}:=\exp \left(\sum_{j=1}^{m} \tau_{j} \int_{z_{0}}^{z} \frac{d z}{z-p_{j}}\right) \quad\left(z \in M_{p_{1}, \ldots, p_{n}}\right)
$$

for some base point $z_{0} \in M_{p_{1}, \ldots, p_{n}}$.

Proof. $d \sigma^{2}$ is reducible only if the image of the representation $\rho_{g}$ is simultaneously diagonalizable, so we may choose a developing map $g: \widetilde{M}_{p_{1}, \ldots, p_{n}} \rightarrow \boldsymbol{C P}^{1}$ such that

$$
\rho_{g}(T)=\left(\begin{array}{cc}
e^{i \theta_{T}} & 0 \\
0 & e^{-i \theta_{T}}
\end{array}\right)
$$

Thus we have

$$
\log \left(g \circ T^{-1}\right)=\log (g)+2 i \theta_{T} .
$$

Differentiating this gives

$$
d \log \left(g \circ T^{-1}\right)=d \log (g),
$$

which implies that $d \log (g)$ is single-valued on $M_{p_{1}, \ldots, p_{n}}$.

On the other hand, by Proposition 4 in [3], there is a complex coordinate $w$ around each end $p_{j}$ such that

$$
a_{j} \star g=\left(w-p_{j}\right)^{\tau_{j}} \quad\left(\tau_{j} \in \boldsymbol{R} \backslash\{0, \pm 1\}\right),
$$

for some $a_{j} \in \mathrm{SU}(2)(j=1, \ldots, n)$. Let $T_{j}$ be the deck transformation of $\widetilde{M}_{p_{1}, \ldots, p_{n}}$ corresponding to a loop surrounding $p_{j}$. Then

$$
\rho_{g}\left(T_{j}\right) \neq \pm \mathrm{id} \quad \text { for } j=1, \ldots, m \text { and } j=n .
$$

Hence $\tau_{j} \notin Z$ when $j \leq m$ and $j=n$. By (B.1), $a_{j}$ in (B.2) is diagonal, so

$$
g\left(p_{j}\right)=0 \quad \text { or } \quad \infty \quad(j=1, \ldots, m, n) .
$$

Hence $d \log (g)$ has poles of order 1 at $p_{1}, \ldots, p_{m}$, and thus

$$
d \log (g)=\frac{d g}{g}=\frac{\tau_{1} d z}{z-p_{1}}+\cdots+\frac{\tau_{m} d z}{z-p_{m}}+u(z) d z
$$

where $u(z)$ is meromorphic. Integrating this gives the assertion.

\section{REFERENCES}

[1] J. L. M. Barbosa and A. G. Colares, Minimal Surfaces in $\boldsymbol{R}^{3}$, Lecture Notes in Math. vol. 1195, Springer-Verlag, (1986).

[2] L. Bieberbach, Theorie Der gewöhnlichen Differentialgleichungen, Zweite Auflage, Springer-Verlag, (1965).

[3] R. Bryant, Surfaces of mean curvature one in hyperbolic space, Astérisque 154-155 (1987), 321-347.

[4] W. Chen and C. Li, What kinds of singular surfaces can admit constant curvature?, Duke Math. J., 78 (1995) 437-451.

[5] C. C. Chen, F. Gackstatter, Elliptische und hyperelliptische Funktionen und vollständige Minimalflächen vom Enneperschen Typ, Math. Ann. 259 (1982), 359-369.

[6] P. Collin, L. Hauswirth and H. Rosenberg, The geometry of finite topology Bryant surfaces, to appear in Ann. of Math.

[7] R. Sa Earp, E. Toubiana, On the geometry of constant mean curvature one surfaces in hyperbolic space, to appear in Illinois J. Math.

[8] R. Sa Earp, E. Toubiana, Meromorphic data for mean curvature one surfaces in hyperbolic space, preprint. 
[9] F. J. Lopez, The classification of complete minimal surfaces with total curvature greater than $-12 \pi$, Trans. Amer. Math. Soc. 334 (1992), 49-74.

[10] R. Osserman, A Survey of Minimal Surfaces, 2nd ed., Dover, 1986.

[11] W. Rossman, K. Sato, Constant mean curvature surfaces with two ends in hyperbolic space, Experimental Math., 7(2) (1998), 101-119.

[12] W. Rossman, M. Umehara and K. Yamada, Irreducible constant mean curvature 1 surfaces in hyperbolic space with positive genus, Tôhoku Math. J. 49 (1997), 449-484.

[13] _ A new flux for mean curvature 1 surfaces in hyperbolic 3-space, and applications, Proc. Amer. Math. Soc. 127 (1999), 2147-2154.

[14] - Mean curvature 1 surfaces in hyperbolic 3-space with low total curvature I, preprint, math.DG/0008015.

[15] _ , Period problems for mean curvature one surfaces in $H^{3}$ (with application to surfaces of low total curvature), preprint.

[16] A. J. Small, Surfaces of Constant Mean Curvature 1 in $H^{3}$ and Algebraic Curves on a Quadric, Proc. Amer. Math. Soc. 122 (1994), 1211-1220.

[17] M. Troyanov, Metric of constant curvature on a sphere with two conical singularities, in "Differential Geometry", Lect. Notes in Math. vol. 1410, Springer-Verlag, (1989), 296-306.

[18] _ Prescribing curvature on compact surfaces with conical singularities, Trans. Amer. Math. Soc. 324 (1991), 793-821.

[19] M. Umehara and K. Yamada, Complete surfaces of constant mean curvature-1 in the hyperbolic 3-space, Ann. of Math. 137 (1993), 611-638.

[20] _ A parameterization of Weierstrass formulae and perturbation of some complete minimal surfaces of $\boldsymbol{R}^{3}$ into the hyperbolic 3-space, J. reine u. angew. Math. 432 (1992), 93-116.

[21] - Surfaces of constant mean curvature-c in $H^{3}\left(-c^{2}\right)$ with prescribed hyperbolic Gauss map, Math. Ann. 304 (1996), 203-224.

[22] _ Another construction of a CMC-1 surface in $H^{3}$, Kyungpook Math. J. 35 (1996), $831-849$

[23] $\ldots$ duality on CMC-1 surface in the hyperbolic 3-space and a hyperbolic analogue of the Osserman Inequality, Tsukuba J. Math. 21 (1997), 229-237.

[24] Metrics of constant curvature one with three conical singularities on the 2-sphere, Illinois J. Math. 44 (2000), 72-94.

[25] Z. Yu, Value distribution of hyperbolic Gauss maps, Proc. Amer. Math. Soc. 125 (1997), 2997-3001.

[26] $575-588$.

(Rossman) Department of Mathematics, Faculty of Science, Kobe University, Rokko, KoBE 657-8501, JAPAN

E-mail address: wayne@math.kobe-u.ac.jp

(Umehara) Department of Mathematics, Faculty of Science, Hiroshima University, Higashi-HiRoshima 739-8526, JAPAN

E-mail address: umehara@math.sci.hiroshima-u.ac.jp

(Yamada) Faculty of Mathematics, Kyushu University 36, 6-10-1 Hakozaki, Higashi-ku, FUKUOKA 812-8185, JAPAN

E-mail address: kotaro@math.kyushu-u.ac.jp 